\title{
FUZZY CONTROL BASED ON CLUSTER ANALYSIS AND DYNAMIC PROGRAMMING
}

\section{Plamena ANDREEVA and George GEORGIEV}

\section{Introduction}

Fuzzy control as an approach to nonlinear and complex control design has attracted a great deal of research interest in the past decade. The basic idea of the approach is to incorporate fuzzy IF-THEN rules into the control design, that is, fuzzy control combines two resources: input-output data and the experts' experience expressed by rules. Therefore, fuzzy control is always applied to the system, which is too complex to get the mathematical model precisely.

Fuzzy control systems are dynamic systems that estimate input - output functions without mathematical model and adaptively infer and generate fuzzy rules. We apply fuzzy control in two general ways. First one is to use only fuzzy controller. The second one is to use fuzzy rules like tuning machine for original controllers.

By complex non-linear objects the control of a process is nothing more than a linguistic model of the human operator strategy and is, as such, a decision model. Individual decision making in fuzzy environment $(\mathrm{FE})$ is:

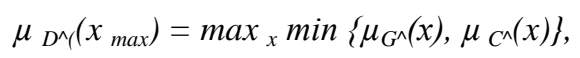

where $x_{\max i}$ is the maximizing decision. Here $\mu_{G^{\wedge}}(x)$ is the fuzzy goal, $\mu_{C^{\wedge}}(x)$ is the fuzzy constrain. We consider the discrete decision space (situations in which there are countable many actions from which the "optimal" has to be chosen). Utilities (cost function) are modeled as fuzzy sets, in the framework of linguistic variables.

\subsection{Problem statement}

The problem of incorporating expert knowledge is wide studied and founds applications in variety of control, signal processing and expert systems. ${ }^{6,10,11}$ It can be considered as non-linear regression to approximate the unknown non-linear 
function based on the input-output data. If the input-output data are measurements of the inputs and outputs of an expert who is demonstrating on how to do a skilled job, then the collection of input-output data is a presentation of the expert's domain knowledge. The advantage of the fuzzy system approach compared with other nonlinear regression methods is that fuzzy systems not only are universal approximators, ${ }^{11}$ but also have clear physical interpretation for their structures and parameters so that the results can be interpreted in terms of fuzzy IF-THEN rules.

The basic problem in fuzzy control application is the analysis of the transmitted uncertainty from the premises to the conclusion. Cluster analysis of the input domain helps to a reasonable extent in the management of uncertainty in such situations.

This paper concerns fuzzy control for autonomous mobile system in 3D space. The associated information system is designed to be a tool for automated analysis and synthesis of closed loops for moving platforms and to store the results from possible training made by an expert and distributed via network. In order to determine the number of rules during the training process a clustering method is proposed. The number of rules is specified by the number of clusters of the input-output data and the parameters are determined from the a priory context dependant knowledge representation.

\subsection{Current research}

Fuzzy models are described by fuzzy rule of type (1) plus some additional parameters. Fuzzy models are just particular instances of the kind of non-linear nonparametric model with the advantage of providing the fuzzy rules as a way to describe some possible available prior knowledge.

$$
\text { If } x_{1} \text { is } A_{1} \text { and } x_{2} \text { is } A_{2} \text { and } \ldots \text { and } x_{n} \text { is } A_{n} \text {, then } y \text { is } B \text {, }
$$

where $A_{1}, A_{2}, \ldots, A_{n}$ and $B_{n}$ are fuzzy sets. The input and output domain of such fuzzy system (shown on Fig.1) are determined in relation to the input and output universe of discourse $U$ of fuzzy sets. A fuzzy set $A$ whose support $A_{\text {sup }}$ is the universe of discourse $U$ with $\mu(x)=1$ is referred to as a fuzzy universe.

The objective of this paper is to perform theoretical analysis of the closed loops for moving objects by the mean of generating clustering method for extracting rules from the Distributed Information System (DIS). The tool for performing this analysis is applied to improve the performance of moving object control and off-line analysis of its efficiency. This research is related to the search of the suitable rule sets of the fuzzy classification system. Clustering method with triangular membership functions is presented and the case with Gaussian membership functions is considered. 


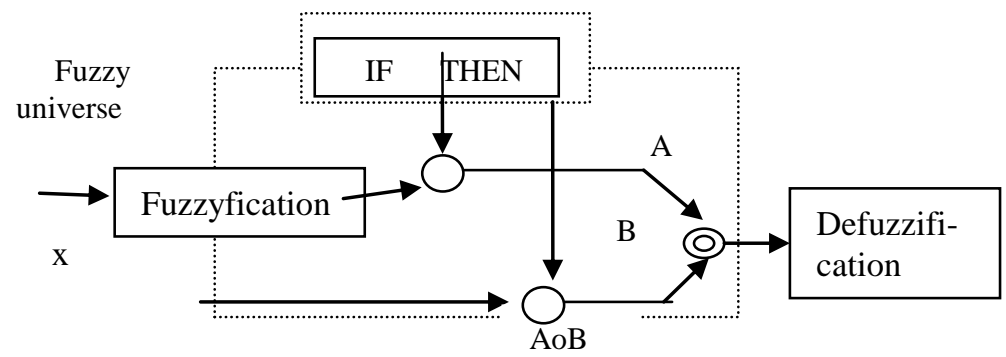

Inference engine

Figure 1. Basic structure of fuzzy control system.

The classification problem is the capability of the system to generate the required decision regions. This process helps in reducing the searching space and time while performing the optimal solution. Because representation of the rule base (R) by an analytical function is unrealistic and infeasible in general, it is customary to assume that the fuzzy sets involved in $\mathrm{R}$ have discrete and finite universes. For reasons of processing efficiency, it is easier to scale the process variables and control signals in fuzzy control algorithm. Real-time modification of a fuzzy rule on the basis of some self-organization scheme is possible. ${ }^{4}$ Our fuzzy approach combines the power of fitting complex data from DIS and the possibility of structuring the knowledge by linguistic rules. More important, the analysis eventually leads to a new representation of the input data.

\section{Analysis of the cluster method}

Classification of a priori knowledge and identification of new data are central in the fuzzy control process. Fuzzy control for moving objects is characterised by model uncertainty and inequality model constraints. The proposed method in (Andreeva, et. al., 2000) of cluster analysis for such a system is used to classify the input data and to receive the rules.

Let us consider the problem as given the input -output data pairs:

$$
\left(x^{p} ; y^{p}\right), \quad p=1,2, \ldots N
$$

where $x^{p} \in U_{x}=\left[\alpha_{1}, \beta_{1}\right] \times \ldots \times\left[\alpha_{n}, \beta_{n}\right] \subset R^{n}, y^{p} \in U_{y}=\left[\alpha_{y}, \beta_{y}\right] \subset R$ and the data are generated by unknown nonlinear function or system $y=f(x)$. Here $\alpha_{i}$ and $\beta_{i}$ are respectively the lower and upper bound of input - output domain, and $U_{i}$ is universe of discourse for variable $x_{i}$. The goal is to design a fuzzy system $f(x)$ based on these $N$ input - output pairs which approximate the unknown function or system $f(x)$. 
If we assume to have two input - output pairs $\left(x^{1} ; y^{1}\right)$ and $\left(x^{2} ; y^{2}\right)$ first we have to define fuzzy sets to cover the input and output spaces. For $i=1,2$ there will be two fuzzy sets $A_{1}{ }^{j}$ and $A_{2}{ }^{j}$, where $j=1,2, \ldots, N$ according to (2).The fuzzy sets have the following membership function:

$$
\begin{gathered}
\mu_{A_{i}^{1}}\left(x_{i}\right)=\mu_{A_{i}^{1}}\left(x_{i} ; e_{i}^{1}, e_{i}^{1}, e_{i}^{2}\right), \\
\mu_{A_{i}^{j_{i}}}\left(x_{i}\right)=\mu_{A_{i} j_{i}}\left(x_{i} ; e_{i}^{j_{i-1}}, e_{i}^{j_{i}}, e_{i}^{j_{i+1}}\right), \\
\mu_{A_{i}^{N_{i}}}\left(x_{i}\right)=\mu_{A_{i} N_{i}}\left(x_{i} ; e_{i}^{N_{i-1}}, e_{i}^{N_{i}}, e_{i}^{N_{i+1}}\right),
\end{gathered}
$$

for $\mathrm{j}_{\mathrm{i}}=2,3, . ., \mathrm{N}_{\mathrm{i}}-1$ and $x_{i}$-input data; $e_{i}^{j i}$ - average value; left boundaries $\mathrm{j}^{\mathrm{i}-1}$ and right boundaries $\mathrm{j}^{\mathrm{i}+1+}$.Here the triangular membership function $\mu \mathrm{A}_{\mathrm{i}}^{\mathrm{j}}$ is defined as :

$$
\mu_{A}\left(x ; a, b, c_{i}\right)=\mid \begin{array}{cc}
\frac{x-a}{b-a}, & a \leq x \leq b \\
\frac{c-x}{c-b}, & b<x \leq c \\
0, & \text { otherwise }
\end{array}
$$

If we assume to have two input - output pairs $\left(x^{l} ; y^{l}\right)$ and $\left(x^{2} ; y^{2}\right)$ first we have to define fuzzy sets to cover the input and output spaces.

$$
\left|x^{k}-x_{c} k^{k}\right| \leq\left|x^{k}-x_{c}^{l}\right|, \quad l=1,2, \ldots, M
$$

For $i=1,2$ there will be two fuzzy sets $A_{1}^{j}$ and $A_{2}^{j}$, where $j=1,2, \ldots, N$ according to (2). The fuzzy sets have the membership function in (3).

Starting with the first input - output pair $\left(x^{l} ; y^{l}\right)$ we establish a cluster center $x_{c}{ }^{l}$ at $x^{l}$ and set $y_{c}{ }^{l}(1)=y^{l}$, where the number of clusters $M=1$ and $y_{c}^{M}$ equals to the centroid of input data. No other cluster exists at this time. For the $k$-th input-output pair $\left(x^{k}\right.$, 
$\left.y^{k}\right), k=2,3, . . K$, there are $M$ clusters with centres at $x_{c}{ }^{l}, x_{c}{ }^{2}, \ldots, x_{c}{ }^{M}$. In the next step find the nearest cluster $x_{c}^{\prime}$ to $x^{k}$.

$$
f_{k}(x)=\frac{\sum_{l=1}^{M} y_{c}^{l}(k) \prod_{j=1}^{2} \mu\left(x_{j} ; x_{c, j}^{l}-r, x_{c, j}^{l}, x_{c, j}^{l}+r\right)}{\sum_{l=1}^{M} \prod_{j=1}^{2} \mu\left(x_{j} ; x_{c, j}^{l}-r, x_{c, j}^{l}, x_{c, j}^{l}+r\right)}
$$

If the absolute value is greater than a selected radius $r$ of first cluster, then establish $x^{k}$ as a new cluster with center $x_{c}{ }^{M+1}=x^{k}$ and set $y_{c}{ }^{M+1}(k)=y^{k}$ and keep $y_{c}^{\prime}(k)=y_{c}^{\prime}(k-1)$. If the absolute value in (5) is less then the radius $r$, than the input point belongs to its closest cluster $k-1$ and compute the cluster's center $y_{c}^{\prime}(k)=y_{c}^{\prime}(k-1)$. The algorithm stops after the reach of last input-output data. We then construct the fuzzy system as:

$$
\left|f(x)-f_{k}(x)\right| \leq k r \sum_{i=1}^{k} \sup \left(\min _{l}\left|x-x_{c}^{l}\right|\right)
$$

In the area outside the cluster the constant value of the nearest cluster center is used as the estimate. This is reasonable because nothing is known about the unknown function in the area not covered by the given data.

This method has no recursion and its simplicity allows soft clustering. It differs from C-Means clustering method ${ }^{3}$ where the number of clusters is predefined. Thus, in contrast to prior research, the applied fuzzy clustering method becomes more suitable for off-line training analysis. The problem is sometimes called unsupervised learning (or self-organization). By unsupervised learning, the performance error on inferring procedure has no effect on the operation of the clustering algorithm. In unsupervised mode, the primary measure that affects the goodness of a cluster is the performance error.

\subsection{Clustering method with triangular membership functions}

We consider the clustering method with assumption that $f(x)$ is continuously differentiable in the whole unit of discourse $U$. If $f_{k}(x)$ is the obtained fuzzy system under the above assumption it holds that : 


$$
\mu_{A_{i}^{j}}\left(x_{i}\right)=\exp \left(-\frac{\left|x_{i}-e_{i}^{j}\right|^{2}}{\sigma^{2}}\right)
$$

in case that $x \in M$, some cluster and $k$ is the number of input-output pairs. The difference between the considered data and the cluster center it belongs to is the Euclidean distance $d_{x}=\min _{\|}\left|x-x_{c}^{\prime}\right|$.

\subsection{Method with Gaussian membership functions}

The complete clustering method was shown in the previous section. Here the method with Gaussian membership function is presented. It is similar to that in section 2.1 with the exception that the membership functions are Gaussian. They are of the form (8).

Then the fuzzy system is constructed as:

$$
f_{k}(x)=\frac{\sum_{l=1}^{M} y_{c}^{l}(k) \exp \left(-\frac{\left|x-x_{c}^{l}\right|^{2}}{\sigma^{2}}\right.}{\sum_{l=1}^{M} \exp \left(-\frac{\left|x-x_{c}^{l}\right|^{2}}{\sigma^{2}}\right.}
$$

Instead of averaged value $y_{c}^{1}$, the linear interpolation of the data inside the cluster can be used to construct fuzzy system. By doing this, more accurate result may be obtained, but the computation is more complex.

\section{Simulation}

The control loops of moving objects were designed on the base of fuzzy control theory. Fuzzy control as an approach to nonlinear and complex control design has attracted a great deal of research interest in the past decade. The autopilot design for flying objects, which are highly nonlinear-coupled system, provides for potentially useful implementation of fuzzy control.

The proposed fuzzy algorithm in section 2.1 is applied to the autonomous mobile system. As input data we consider the experimental data collected during the training process. For modeling dynamic characteristics of moving objects we use at least six nonlinear equations which describe linear and angular positions of rigid body in 3D space with 6 degrees of freedom. Vehicle parameters (geometric and cinematic) are 
space vector $\left(x_{n}, y_{n}, z_{n}\right)$, velocity $v$, control angle $\theta$ and its azimuth $\alpha$. The output variables are the control angle $\varphi$ and vehicle velocity $v_{n}$.

The altitude, airspeed, vertical speed and angular position are measured. The control of the aeroplane is done by IF-THEN rules. Fuzzy control combines two resources: input-output data and the experts' experience expressed by rules. The a priori information is given in the initial state of input data. The universe of discourse of angle $\varphi$ is chosen $[0,360]$ and velocity $v_{n}$ is $[0,980]$. For the partitioning of input data we assume that the fuzzy sets of $\varphi$ and $v_{n}$ have symmetric triangular membership functions.

The fuzzy clustering method is performed as follow:

1) For each input variable $X_{i}$ and output $\varphi$ and $v_{n}$ we define fuzzy sets to cover the input and output spaces.

For the 3 input variable we define $N_{1}, N_{2}$ and $N_{3}$ fuzzy sets $A_{1}{ }^{j}, A_{2}{ }^{j}, A_{3}{ }^{j}$ $\left(\mathrm{j}=1,2, . . \mathrm{N}_{\mathrm{i}}\right)$ on each $\left[\alpha_{\mathrm{i}}, \beta_{\mathrm{i}}\right]$ with the triangular membership functions as in (3).

2) From one input-output pair we generate then one rule.

For each input variable determine the fuzzy set $\mathrm{A}_{1}{ }^{\mathrm{j}}, \mathrm{A}_{2}{ }^{\mathrm{j}}, \mathrm{A}_{3}{ }^{\mathrm{j}}$ in which $v^{\mathrm{p}}, \theta^{\mathrm{p}}$ and $\alpha^{p}\left(p=1,2, \ldots N_{i}\right)$ has the largest membership value. Similarly define $v_{n}{ }^{p}$ and $\varphi^{p}$ such that $\mu v_{\mathrm{n}}{ }^{\mathrm{p}}\left(\mathrm{y}^{\mathrm{p}}\right)>=\mu v_{\mathrm{n}}\left(\mathrm{y}^{\mathrm{p}}\right)$ and $\mu \varphi^{\mathrm{p}}\left(\mathrm{y}^{\mathrm{p}}\right)>=\mu \varphi\left(\mathrm{y}^{\mathrm{p}}\right)$ for $\mathrm{p}=1,2, \ldots \mathrm{N}_{\mathrm{y}}$. Finally the fuzzy IF- THEN rule is:

$$
\begin{aligned}
& \text { If } v \text { is } A_{1}{ }^{j} \text {, and } \theta \text { is } A_{2}{ }^{j} \text {, and } \alpha \text { is } A_{3}{ }^{j,} \\
& \text { THEN } v_{n} \text { is } B_{1}{ }^{p} \text { and } \varphi \text { is } B_{2}{ }^{p}
\end{aligned}
$$

3) Degrees are assigned to each rule generated in 2).

If there exist conflicting rules (with the same IF parts but different THEN parts) then assign a degree to each rule generated in 2).

$$
\operatorname{Deg}(\text { rule })=\Pi_{i} \mu_{A i}\left(\nu^{p}, \theta^{p} \text { and } \alpha^{p}\right) \cdot \mu_{B j}\left(v_{n}{ }^{p}, \varphi^{p}\right)
$$

where $\mathrm{i}=1,2,3$, and $\mathrm{j}=1,2$. Then keep only one rule from a conflict group that has the maximum degree.

4) The fuzzy rule base $(\mathrm{R})$ will be created as :

Rule $^{(\mathrm{f})}$ : If $x_{1}$ is $A_{1}{ }^{j 1}$, and $x_{2}$ is $A_{2}{ }^{j 2}$, and $x_{3}$ is $A_{3}{ }^{j 3}$,

$$
\text { THEN } y \text { is } B^{(f)}
$$

with $f$ index set of the rule base.

The condition $\Sigma \mu_{j c}=1$, where $i$ is i-th input data and $c$ is number of the cluster $i$ belongs to, is not satisfied. This follows from the interpretation of membership values in the $c$-th partitioning. The boundaries between the classes are really fuzzy - more data belong to one cluster with different $\mu$. 


\section{Algorithm and decision-making method}

An algorithm for fuzzy control of an autonomous mobile system in 3D space is described. The algorithm is synthesized on the basis of the Bellman and Zadeh decision-making method in vague environment. The introduction of a goal and a constraint are presented as fuzzy sets. The autonomous vehicle attains the goal by avoiding the constraint by dynamic programming in fuzzy environment. The coordinates of the model movement are determined by maximum-minimum composition. A maximizing decision is defined as an intersection in the space of alternatives of membership functions of a fuzzy goal and a fuzzy constraint at which the membership function of a fuzzy decision attains its maximum.

\subsection{Initial Conditions}

The problem involves finding the best possible time (optimal) registration path of the autonomous mobile system from the initial state to the goal by avoiding the constraint. The goal and the constraint do not change theirs coordinates during the process of decision making. The goal is presented by as a physical previous given point at which the autonomous mobile system has to land or cross. The constraint is presented as an object, which the autonomous mobile system has to avoid. It is given the following initial conditions:

$\mathrm{x}_{0}, \mathrm{y}_{0} \quad$ - coordinates of the initial state

$\mathrm{x}_{\mathrm{g}}, \mathrm{y}_{\mathrm{g}} \quad$ - final coordinates

$\mathrm{x}_{\mathrm{c}}, \mathrm{y}_{\mathrm{c}} \quad$ - coordinates of the constraint

$v_{\text {OK }} \quad$ - average initial velocity value of the model by air

$\gamma_{\mathrm{ok}} \quad$ - average initial value of the model flight-path angle

$\Psi_{\mathrm{ok}} \quad$ - initial model azimuth angle by north

$\mathrm{h}_{\mathrm{ok}} \quad$ - initial altitude of the goal

$\Delta \Psi \quad$ - increment of the model azimuth angle

$\Delta \Phi \quad$ - increment of the model bank angle

$\Delta \mathrm{t} \quad$ - increment of the real time of movement

$\Phi_{\max }$ - maximum value of the model bank angle

$\mathrm{w}_{\mathrm{X}} \quad$ - wind acceleration along the $\mathrm{x}$-axis

$\mathrm{w}_{\mathrm{y}} \quad$ - wind acceleration along the $\mathrm{y}$-axis

L $\quad-$ lift

D $\quad-$ drag

m - mass 


$$
\begin{array}{ll}
g & \text { - acceleration of gravity } \\
\rho & \text { - air density } \\
\mathrm{s} & \text { - model reference area } \\
\alpha & \text { - angle of attack } \\
\alpha_{0} & \text { - zero lift angle } \\
\mathrm{C}_{\mathrm{D}}, \mathrm{C}_{\mathrm{D} 0} & \text { - drag coefficient and zero-lift drag coefficient } \\
\mathrm{C}_{\mathrm{L}}, \mathrm{C}_{\mathrm{L} \alpha} \text { - lift coefficient and its partial derivative of } \alpha \\
\text { wins } & \text { - width of the matching window } \\
\mathrm{k}_{\mathrm{X}}, \mathrm{k}_{\mathrm{y}} & \text { - coefficient reflecting the slope of the selected membership functions }
\end{array}
$$

The mass of the autonomous mobile system is concentrated in one point. The flexible structure of the model is described by the function $f^{L}(v, \gamma, \Phi)$ and $f^{D}(v, \gamma, \Phi)$. The model of the autonomous mobile system is described by the following nonlinear derivative equations:

$$
\begin{aligned}
& \dot{v}_{t}=\frac{-D}{m_{t}}-g \cdot \sin \gamma_{t}-w_{x} \cdot \cos \gamma_{t}-w_{h} \cdot \sin \gamma_{t} \\
& \dot{\gamma}_{\boldsymbol{t}}=\frac{\boldsymbol{L}}{\boldsymbol{m}_{\boldsymbol{t}} v_{\boldsymbol{t}}} \cos \Phi-\frac{\boldsymbol{g}}{v_{t}} \cos \gamma_{\boldsymbol{t}}-\frac{\left(\boldsymbol{w}_{\boldsymbol{x}} \cdot \sin \gamma_{\boldsymbol{t}}-\boldsymbol{w}_{\boldsymbol{y}} \cdot \cos \gamma_{\boldsymbol{t}}\right)}{v_{\boldsymbol{t}}} \\
& \dot{\Psi}_{t}=\frac{L}{m_{t} v_{t} \cos \gamma_{t}} \cdot \sin \Phi \\
& \dot{\boldsymbol{x}}_{\boldsymbol{t}}=v_{t} \cos \gamma_{t} \cos \Psi_{t} \\
& \dot{\boldsymbol{y}}_{\boldsymbol{t}}=v_{t} \cos \gamma_{t} \sin \Psi_{t} \\
& \dot{h}_{t}=v_{t} \sin \gamma_{t}
\end{aligned}
$$

Where: $\quad L=\frac{1}{2} \rho \cdot v_{t}^{2} \cdot s_{t} \cdot C_{L} \cdot f^{L}(v, \gamma, \Phi)$

$$
\begin{aligned}
& C_{L}=C_{L \alpha}\left(\alpha-\alpha_{0}\right) \\
& D=\frac{1}{2} \rho \cdot v_{t}^{2} \cdot s_{t} \cdot C_{D} \cdot f^{D}(v, \gamma, \Phi)
\end{aligned}
$$


The system of derivative equations is integrated by Oilier. Thus the following system of recurrent equations is obtained:

$$
\begin{aligned}
& v_{k+1}=v_{k}-\frac{D}{m} \cdot \Delta t-\left(g \cdot \sin \gamma_{k}+w_{x} \cdot \cos \gamma_{k}+w_{y} \cdot \sin \gamma_{k}\right) \cdot \Delta t \\
& \gamma_{k+1}=\gamma_{k}+\frac{L \cdot \cos \Phi}{m \cdot v_{k}} \cdot \Delta t-\frac{g \cdot \cos \gamma_{k}-w_{x} \cdot \sin \gamma_{k}+w_{y} \cdot \cos \gamma_{k}}{v_{k}} \cdot \Delta t \\
& \Psi_{k+1}=\Psi_{k}+\frac{L}{m \cdot v_{k} \cdot \cos \gamma_{k}} \cdot \sin \Phi \cdot \Delta t \\
& x_{k+1}=x_{k}+v_{k} \cdot \cos \gamma_{k} \cdot \cos \Psi_{k} \cdot \Delta t \\
& y_{k+1}=y_{k}+v_{k} \cdot \cos \gamma_{k} \cdot \sin \Psi_{k} \cdot \Delta t \\
& h_{k+1}=h_{k}+v_{k} \cdot \sin \gamma_{k} \cdot \Delta t
\end{aligned}
$$

The states of the system are $\mathrm{x}_{\mathrm{k}}, \mathrm{y}_{\mathrm{k}}, v_{\mathrm{k}}, \mathrm{h}_{\mathrm{k}}, \Psi_{\mathrm{k}}, \gamma_{\mathrm{k}}$. The system can be controlled by the control angle $\Phi_{\mathrm{k}}$. and the velocity $v_{\mathrm{k}}$. The dynamic features of the object may be given by limitations of the control angle $\Phi_{\mathrm{k}}$ depending on its velocity $v_{\mathrm{k}}$. The limitations are described for a specific case as follows:

$$
\Phi=\left\{\begin{array}{c}
\Phi_{\text {max }}, \Phi \geq \Phi \text { max }_{\text {max }}<\Phi<\Phi_{\text {max }} \\
\Phi,-\Phi_{\text {max }}<\Phi{ }_{\text {max }}, \Phi \leq-\Phi \text { max }
\end{array}\right.
$$

where: $\Phi_{\max }=-55^{\circ} \cdot \frac{v}{10} ; \quad+55^{\circ} \cdot \frac{v}{10}$

The membership function of the fuzzy goal might be presented as an attractive function, which has a maximum at the final point. Its kind is described as follows:

$$
\mu_{g}(x, y)=e^{-k_{x}\left(x_{g}-x\right)^{2}-k_{y}\left(y_{g}-y\right)^{2}}
$$

The membership function of the fuzzy constraint might be presented as a repulsive function and it is described by the following form:

$$
\mu_{c}(x, y)=1-e^{-k_{x}\left(x_{c}-x\right)^{2}-k_{y}\left(y_{c}-y\right)^{2}}
$$


In the algorithm the following variables and areas are used:

$\mathrm{i}, \mathrm{j}, \mathrm{k}, \min , \max$ - work variables

$\mathrm{x}_{\mathrm{k}}$ (wins) - work area of possible $\mathrm{x}$-coordinates of the movement trajectory

$\mathrm{y}_{\mathrm{k}}$ (wins) - work area of possible $\mathrm{y}$-coordinates of the movement trajectory

$\Psi_{\mathrm{k}}$ (wins) - work area of possible azimuth angles of the movement trajectory

$\mu_{\mathrm{g}}$ (wins) - work area of the membership function values of the model states by the goal

$\mu_{\mathrm{c}}$ (wins) - work area of the membership values of the model states by the constraint

$\mathrm{i}_{\max } \quad$ - variable for the width of the matching window at which the system states is obtained maximizing intersection

$v_{\mathrm{k}}, v_{\mathrm{ok}} \quad$ - variables for the model velocity

$\gamma_{\mathrm{k}}, \gamma_{\mathrm{ok}} \quad$ - variables for the model flight-path angle

$\mathrm{h}_{\mathrm{k}}, \mathrm{h}_{\mathrm{ok}} \quad$ - variable for the model altitude

$\mathrm{x}_{\mathrm{Ok}}$

- variable for the model $\mathrm{x}$-coordinate

$\mathrm{y}_{\mathrm{ok}}$

- variable for the model y-coordinate

$\Psi_{\mathrm{ok}}$

- variable for the model azimuth angle

$\mathrm{K}$

- number of the one-stage decision process

$\mathrm{x}_{\mathrm{tr}}(\mathrm{K}) \quad$ - work area of optimal $\mathrm{x}$-coordinates for the whole movement trajectory

$y_{\operatorname{tr}}(K) \quad$ - work area of optimal y-coordinates for the whole movement trajectory

$\Psi_{\mathrm{tr}}(\mathrm{K}) \quad$ - work area of optimal azimuth angles for the whole movement trajectory

$\Phi_{\mathrm{c}} \quad$ - variable for the control value of the bank angle which has been computed for the optimal azimuth angle

$\Psi_{\mathrm{t}} \quad$ - variable for the current computed azimuth angle

$\mathrm{d} \Psi_{\mathrm{t}} \quad$ - variable for the current module difference of the azimuth angle

$\mathrm{d} \Psi_{\min } \quad$ - variable for the minimum module difference of the azimuth angle

Subscripts:

$\mathrm{K}$ - new value of the model states of the decision process

o, ok - initial or old value of the model states of the decision process

$\mathrm{t}$ - current value 


\subsection{Control Algorithm}

\section{Step 1}

It is given the following initial conditions:

$\mathrm{x} 0, \mathrm{y} 0$ - coordinates of the initial state

$\mathrm{xg}, \mathrm{yg}$ - final coordinates

$\mathrm{xc}, \mathrm{yc}$ - coordinates of the constraint

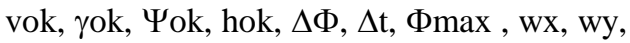

$\mathrm{L}, \mathrm{D}$,

$\mathrm{m}, \mathrm{g}, \rho, \mathrm{s}, \alpha, \alpha 0, \mathrm{C}_{\mathrm{D}}, \mathrm{C}_{\mathrm{D} 0}, \mathrm{C}_{\mathrm{L}}, \mathrm{C}_{\mathrm{L} \alpha}$, wins,

$\mathrm{kx}, \mathrm{ky}$

$$
\Delta \Psi=\frac{2 . \pi}{\operatorname{wins}}, x_{o k}=x_{g}, y_{o k}=y_{g}, k=1
$$

\section{Step 3}

$x_{k}(i)=x_{o k}-v_{o k} \cdot \cos \gamma_{o k} \cdot \cos \left(\Psi_{o k}\right) \cdot \Delta t$

$y_{k}(i)=y_{o k}-v_{o k} \cdot \cos \gamma_{o k} \cdot \sin \left(\Psi_{o k}\right) \cdot \Delta t$

$\mu_{g}(i)=e^{-k_{x}\left(x_{0}-x_{k}(i)\right)^{2}-k_{y}\left(y_{0}-y_{k}(i)\right)^{2}}$

$\mu_{c}(i)=1-e^{-k_{x}\left(x_{c}-x_{k}(i)\right)^{2}-k_{y}\left(y_{c}-y_{k}(i)\right)^{2}}$

\section{Step 5}

$\mathrm{I}=1 \quad \mathrm{Max}=0$

\section{Step 7}

IF $(\min \geq \max )$ THEN $\max =\min$

$\mathrm{I}_{\max }=\mathrm{j}$

\section{Step 9}

$$
\begin{aligned}
& x_{t r}(k)=x_{k}\left(i_{\max }\right), y_{t r}(k)=y_{k}\left(i_{\max }\right), \\
& \Psi_{t r}(k)=\Psi_{k}\left(i_{\max }\right), x_{o k}=x_{k}\left(i_{\max }\right), \\
& y_{o k}=y_{k}\left(i_{\max }\right), \Psi_{o k}=\Psi_{k}\left(i_{\max }\right)
\end{aligned}
$$

\section{Step 2}

$\mathrm{i}=1$

$\Psi_{k}(i)=\Psi_{o k}$

\section{Step 4}

IF ( $\mathrm{i} \neq$ wins $)$ THEN

$\mathrm{i}=\mathrm{i}+1$

$\Psi_{k}(i)=\Psi_{k}(i-1)+\Delta \Psi$

IF $\left(\Psi_{k}(i)>2 . \pi\right)$ THEN

$$
\Psi_{k}(i)=\Psi_{k}(i)-2 . \pi
$$

$$
\Psi_{o k}=\Psi_{k}(i)
$$

go to Step 3

\section{Step 6}

IF $\left(\mu_{g}(i) \leq \mu_{c}(i)\right)$ THEN

$$
\begin{aligned}
& \min =\mu_{g}(i) \\
& \mathrm{j}=\mathrm{I} \\
& \min =\mu_{c}(i) \\
& \mathrm{j}=\mathrm{I}
\end{aligned}
$$

\section{ELSE}

\section{Step 8}

IF ( $\mathrm{i} \neq$ wins) THEN $\mathrm{i}=\mathrm{i}+$ 1 go to Step 6

\section{Step 10}

IF $\left(\left(\mathrm{x}_{\mathrm{ok}} \neq \mathrm{x}_{0}\right)\right.$ or $\left.\left(\mathrm{y}_{\mathrm{ok}} \neq \mathrm{y}_{0}\right)\right)$

THEN $\mathrm{k}=\mathrm{k}+1$ go to 


\section{Step 11}

$$
\begin{aligned}
& \Psi_{t}=\frac{L}{m \cdot v_{o k} \cdot \cos \gamma_{o k}} \cdot \sin \left(-\Phi_{\text {max }}\right) \cdot \Delta t \\
& d \Psi_{\text {min }}=\left|\Psi_{t}-\Psi_{t r}(k)\right| \\
& \Phi_{c}=-\Phi_{\text {max }}, \Phi=-\Phi_{\text {max }}+\Delta \Phi
\end{aligned}
$$$$
\text { Step } 13 \text { IF }\left(d \Psi_{t} \leq d \Psi_{\min }\right)
$$$$
d \Psi_{\min }=d \Psi_{t} \quad \Phi_{c}=\Phi
$$

\section{THEN Step 14}$$
\text { go to Step } 12
$$

\section{Step 15}

$$
\begin{aligned}
& \gamma_{k}=\gamma_{o k}+\frac{L \cdot \cos \Phi_{c}}{m \cdot v_{o k}} \cdot \Delta t- \\
& \frac{g \cdot \cos \gamma_{o k}+w_{x} \cdot \sin \gamma_{o k}-w_{y} \cdot \cos \gamma_{o k}}{v_{o k}} \cdot \Delta t \\
& v_{k}=v_{o k}-\frac{D}{m} \cdot \Delta t-\left(g \cdot \sin \gamma_{o k}+\right. \\
& \left.w_{x} \cdot \cos \gamma_{o k}+w_{y} \cdot \sin \gamma_{o k}\right) \cdot \Delta t \\
& h_{k}=h_{o k}+v_{o k} \cdot \sin \gamma_{o k} \cdot \Delta t \\
& \gamma_{o k}=\gamma_{k}, v_{o k}=v_{k}, h_{o k}=h_{k}
\end{aligned}
$$

The model movement is directed by the calculated bank angle $\Phi_{\mathrm{c}}, \mathrm{x}_{\mathrm{Ok}}, \mathrm{y}_{\mathrm{Ok}}, v_{\mathrm{ok}}$, $\mathrm{h}_{\mathrm{ok}}, \Psi_{\mathrm{ok}}$, and $\gamma_{\mathrm{ok}}$.
IF $\left(\Phi \neq \Phi_{\max }\right)$ THEN

$$
\Phi=\Phi+\Delta \Phi
$$

Step 12

$$
\Psi_{t}=\frac{L}{m \cdot v_{o k} \cdot \cos \gamma_{o k}} \cdot \sin (\Phi) \cdot \Delta t
$$$$
\text { Step } 16
$$$$
\text { IF }(k \neq 0) \text { THEN }
$$$$
\mathrm{k}=\mathrm{k}-1
$$

ELSE go to Step 11

\section{End}

\subsection{Description of the Algorithm}

The decision uses the method of dynamic programming in fuzzy environment. The width value of the matching window is a variable named wins. The fuzzy environment consists of a goal and a constraint. The matched pairs of membership function values of the goal and the constraint are compared in the range of the matching window. Finding the best time (optimal) registration path of the model is connected with the decision of the system of nonlinear derivative equations. The purpose of the presented algorithm is to demonstrate a fuzzy method for determination of the trajectory of the dynamic object, which is described by nonlinear derivative equations. The problem of finding the optimal registration path of the model is a multistage decision process. The original multistage ( $\mathrm{K}$ - stage) decision process is replaced as $\mathrm{K}$ one-stage processes. It is the principle of optimality on which dynamic programming is based. That is why the model equations (22) - (27) are presented in a recurrent form. The model state a for $\mathrm{k}+1$ one-stage decision process may be expressed in the following way: 


$$
\begin{aligned}
& \mathrm{X}_{k+1}=\mathrm{X}_{k}+\operatorname{Max}\left(\mu_{g}(1) \wedge \mu_{c}(1), \mu_{g}(2) \wedge\right. \\
& \left.\mu_{c}(2), \ldots, \mu_{g}(\text { wins }) \wedge \mu_{c}(\text { wins })\right)
\end{aligned}
$$

where:

$\mathrm{X}_{k}$ - general symbol for the model state $\left(\mu_{g}(i), \mu_{c}(i)\right)$ - matched pair of membership function values of the goal and the constraint $1 \leq \mathrm{i} \leq$ wins - range of alteration

In the beginning the algorithm uses the reverse problem of dynamic programming. The model is moved from the goal to the initial state. The optimal movement trajectory is calculated in an Off-line mode. The next initial condition is admitted: The model velocity $v_{\mathrm{ok}}$ and the model flight-path angle $\gamma_{\mathrm{ok}}$ accept an average initial value. Equations (25) and (26) are used. The model azimuth angle is altered from 0 to $2 \pi$ for each one-stage decision process. The increment of the azimuth angle $\Delta \Psi$ is calculated by the following expression:

$$
\Delta \Psi=\frac{2 . \pi}{\text { wins }}
$$

The pair coordinates $\mathrm{x}_{\mathrm{k}}(\mathrm{i})$ and $\mathrm{y}_{\mathrm{k}}(\mathrm{i})$ are calculated. It begins with the given initial azimuth angle $\Psi_{\mathrm{ok}}$ :

$$
\begin{aligned}
& x_{k}(i)=x_{o k}-v_{o k} \cdot \cos \gamma_{o k} \cdot \cos \left(\Psi_{o k}\right) \cdot \Delta t \\
& y_{k}(i)=y_{o k}-v_{o k} \cdot \cos \gamma_{o k} \cdot \sin \left(\Psi_{o k}\right) \cdot \Delta t
\end{aligned}
$$

The calculated pair coordinates are replaced in the membership functions of the fuzzy goal and the fuzzy constraint. The possible coordinates of the next point from the movement trajectory and the corresponding azimuth angles are stored in the three area $\mathrm{x}_{\mathrm{k}}$ (wins), $\mathrm{y}_{\mathrm{k}}$ (wins) and $\Psi_{\mathrm{k}}$ (wins). By using a maximum-minimum composition such model coordinates are determined at which the intersection of matched pairs of membership functions of the goal and the constraint obtains maximum value. Those values of the azimuth angles are stored at which the coordinates get maximum value. This is being done for each $\mathrm{k}$ one-stage decision process. The policy function is determined on the basis of the azimuth angle by maximum-minimum composition and it is expressed as follows:

$$
\Psi_{o k}=\pi\left(\operatorname{Max}\left(\mu_{g}\left(i_{\max }\right) \wedge \mu_{c}\left(i_{\max }\right)\right)=\pi\left(\operatorname{Max}\left(\mu_{g \wedge c}\left(i_{\max }\right)\right)\right)=\Psi_{k}\left(i_{\max }\right)\right.
$$

where:

$\mu_{g}\left(i_{\max }\right)=e^{-k_{x}\left(x_{0}-x_{k}\left(i_{\max }\right)\right)^{2}-k_{y}\left(y_{0}-y_{k}\left(i_{\max }\right)\right)^{2}}$ - is the membership function value by the goal at which the system state has obtained a maximizing intersection 
$\mu_{c}\left(i_{\max }\right)=1-e^{-k_{x}\left(x_{c}-x_{k}\left(i_{\max }\right)\right)^{2}-k_{y}\left(y_{c}-y_{k}\left(i_{\max }\right)\right)^{2}}$ - is the membership function value by the constraint at which the system state has obtained a maximizing intersection $\mathrm{i}_{\max }$ - is the width value of the matching window at which the system state has obtained a maximizing intersection.

The optimal coordinates of the movement trajectory and the corresponding angle of the azimuth are stored in three areas $x_{t r}(k), y_{t r}(k)$ and $\Psi_{t r}(k)$. Their maximum dimension is equal to the number of the one-stage decision processes $K$. The algorithm uses the direct problem of dynamic programming in an On-line mode after it has attained the initial state. The bank angle is altered from $\Phi_{\max }$ to $\Phi_{\max }$ in equation (24) for each one-stage process. The average initial values of the model velocity $v_{\mathrm{ok}}$ and the flight-path angle $\gamma_{\mathrm{ok}}$ are substituted for the first calculation of the azimuth angle $\Psi_{\mathrm{t}}$. The difference by module $\mathrm{d} \Psi_{\min }$ is formed between the calculated azimuth angle $\Psi_{\mathrm{t}}$ and the calculated azimuth angle from the reverse problem of dynamic programming $\Psi_{\mathrm{tr}}(\mathrm{k})$. The minimum module difference $\mathrm{d} \Psi_{\min }$ is looked for each alteration of the increment of the bank angle $\Delta \Phi$. This value of the bank angle $\Phi_{\mathrm{c}}$ is stored at which the module difference $\mathrm{d} \Psi_{\text {min }}$ has obtained minimum value. The calculated bank angle $\Phi_{c}$ is replaced in equation (23) and the new flight-path angle is calculated. The new model velocity and altitude are calculated in equations (22) and (27). The system states are found. The problem of finding the best possible time (optimal) registration path of autonomous mobile system is decided. The model movement is directed by the calculated bank angle $\Phi_{\mathrm{c}}$.

\section{Conclusion}

This paper focuses on fuzzy control of a class of nonlinear systems, which are characterized by model uncertainty and inequality model constraints. It deals with an autonomous moving system for control of flying objects and a distributed information system, designed as a tool for automated analysis and synthesis of closed loop. Different clustering methods are explored and compared. The associated Intelligent Information System (IIS) is designed to store the results from possible training made by an expert and distributed via network. The paper considers cluster analysis for such a system, based on Bezdek's fuzzy cluster method (FCM). The proposed method is used to classify the input data and to receive the rules.

The number of rules is specified by the number of clusters. It is used to classify the input data and to receive the rules for DIS. This rules can then be used for the design of rule-based intelligent systems. When a new unknown input is added the fuzzy classifier scheme proceeds and executes the rule to find a new fact. This allows stepwise refinement in DIS. We have discussed a possible analysis toward this objective. At the end some simulation results are given. The results showed that the 
key factors for accuracy are the resolution of the input space covering and the a priori information.

This has to be further explored. In addition, it would be interesting to develop statistical methods for diagnostics and learning from fuzzy - neural nets. Then the individual fuzzy rules can be learned using an error back-propagation algorithm.

An example of fuzzy control for autonomous mobile system in 3D space is explored and the results from the decision using the method of dynamic programming in fuzzy environment are shown. The synthesized algorithm guides an autonomous vehicle in 3D space which pursues an object and evades an obstacle. The fuzzy control is based on determination of a maximizing decision by using dynamic programming. The maximizing decision is defined as a point in the space of alternatives at which the membership function of a fuzzy decision attains its maximum value. The purpose of the presented algorithm is to demonstrate a fuzzy method for determination of the trajectory of the dynamic object.

A system of functional equations can be decided by using dynamic programming and appropriate membership functions for fuzzy environment. The optimal registration path is computed for an autonomous mobile system in 3D space. The fuzzy control has been presented for model movement along the optimal path. In this sense the above-described approach can be used for some other functional applications as well. The fuzzy environment could be expanded, too.

\section{Acknowledgement}

The work on this paper was supported by the Bulgarian Academy of Sciences, project "Development of Moving Platform simulator".

\section{References}

1. Andreeva, P., N. Tchingov and R. Michel, "Cluster Analysis for Fuzzy Control Systems," Proc. of IFAC Conference CSD'2000 (Bratislava, Slovak Republic, 12-15 June 2000).

2. Bellman, R.E. and L.A. Zadeh, "Decision making in fuzzy environment," Management science 17, 4 (1970), 141-164.

3. Bezdek J.C. and Pal S.K, Fuzzy Models for Pattern Recognition (NJ: IEEE Press, 1992).

4. Driankov D. and H. Hellendoorn, An Introduction to fuzzy control (Berlin: Springer Verlag, 1997).

5. Georgiev, G., "Algorithm for fuzzy control of autonomous mobile robot," INCON'97 (Sofia, Bulgaria, October 13-15, 1997), 53-56.

6. Hirota, K., ed., Industrial Applications of Fuzzy Technologies (Tokyo: Springer Verlag, 1994). 
7. Kubota, T. and H. Hashimoto, "A strategy for collision avoidance among moving obstacle for a mobile robot," IFAC Control Conference (1992), Vol. 5, pp. 103-108.

8. Penev, V., "Heurestic Decision-Making Training System," EUFIT'94 (Aachen, Germany, September 20-23, 1994), Vol. 2, pp.660-664.

9. Sakoe H. and S. Chiba, "A dynamic programming approach to continuous speech recognition," Proc. Int. Congres on Acoustics (Budapest, Hungary, 1971), Paper 20, C13.

10. Wang, L.-X., Adaptive fuzzy Systems and Control (Englewood Cliffs, N.J.: Prentice Hall, 1994).

11. Wang, L.-X. and L.M. Mendel, "Generating Fuzzy Rules by Learning from Examples," IEEE Trans. on Systems, Man and Cybernetics 22 (1992), 1414-1427

PLAMENA TSANOVA ANDREEVA holds a M.Sc. degree in electronics from the Technical University of Sofia, Bulgaria (1986), and graduated the Informatics course in Theoretical Computer Science at the Institute of Informatics from the University of Fribourg, CH (1998). She is interesting in Knowledge Based Systems and Artificial Intelligence. IEEE , EWISH member. Mrs. Andreeva is currently a research associate at the Department of Knowledge Based Control Systems, Institute of Control and System Research of the Bulgarian Academy of Sciences. E-mail: plamena@iusi.bas.bg.

GEORGE LUBOMIROV GEORGIEV: M.Sc.(1985, Computer Technologies) from the Technical University of Sofia, Bulgaria. Research and design works in the field of intelligent technologies engineering (signal processing, fuzzy control, fuzzy reasoning, neuro-like structures); Projects of robot control systems; sensors \& systems based on modern control theory; simulators. Mr. Georgiev is currently a research associate at the Department of Knowledge Based Control Systems, Institute of Control and System Research of the Bulgarian Academy of Sciences. E-mail: joro@iusi.bas.bg. 